\title{
Corticosteroid injection or dry needling for musculoskeletal pain and disability? A systematic review and GRADE evidence synthesis
}

\author{
Luis Fernando Sousa Filho ${ }^{1,2^{*}}$ (1), Marta Maria Barbosa Santos ${ }^{2}$, Gabriel Henrique Freire dos Santos ${ }^{2}$ and \\ Walderi Monteiro da Silva Júnior ${ }^{1,2}$
}

\begin{abstract}
Background: Corticosteroid injection and dry needling have been used in the treatment of musculoskeletal conditions, but it is unclear which intervention is the most effective. The purpose of this study was to compare the effects of corticosteroid injection and dry needling for musculoskeletal conditions at short-, medium-, and long-term follow-up.

Methods: Electronic databases were searched up to 31 October 2021. Two researchers independently screened titles, abstracts and full-text articles. Randomized clinical trials (RCTs) that investigated the effectiveness of dry needling compared to corticosteroid injection in patients over 18 years with a musculoskeletal condition were included in the review. The studies had to report pain and/or disability as outcome. Risk of bias was assessed by using the revised Cochrane Collaboration tool (RoB 2.0). Quality of evidence was evaluated by using the GRADE approach.
\end{abstract}

Results: Six studies were included ( $n=384$ participants). Four musculoskeletal conditions were investigated. There is very low-quality evidence that CSI is superior to DN for reducing heel pain (plantar fasciitis) and lateral elbow pain at short- and medium-term follow-up, but not for myofascial pain and greater trochanteric pain. There is very low-quality evidence that DN is more effective than CSI at long-term follow-up for reducing pain in people with plantar fasciitis and lateral epicondylitis. Very low-certainty evidence shows that there is no difference between DN and CSI for disability at short-term follow-up. One study showed that CSI is superior to DN at medium-term follow-up and another observed that DN is superior to CSI for reducing disability at long-term.

Conclusions: There are no differences between DN and CSI in pain or disability for myofascial pain and greater trochanteric pain syndrome. Very-low certainty evidence suggests that CSI is superior to DN at shorter follow-up periods, whereas DN seems to be more effective than CSI at longer follow-up durations for improving pain in plantar fasciitis and lateral epicondylitis. Large RCTs with higher methodological quality are needed in order to draw more incisive conclusions.

PROSPERO registration number: CRD42020148650.

\footnotetext{
${ }^{*}$ Correspondence: fernandosouf@hotmail.com

${ }^{1}$ Graduate Program in Physical Education, Federal University of Sergipe,

Av Marechal Rondon, s/n, Rosa Elze, São Cristovão, Sergipe 49100-000,

Brazil

Full list of author information is available at the end of the article
}

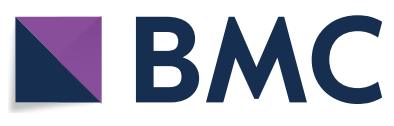

The Author(s) 2021. Open Access This article is licensed under a Creative Commons Attribution 4.0 International License, which permits use, sharing, adaptation, distribution and reproduction in any medium or format, as long as you give appropriate credit to the original author(s) and the source, provide a link to the Creative Commons licence, and indicate if changes were made. The images or other third party material in this article are included in the article's Creative Commons licence, unless indicated otherwise in a credit line to the material. If material is not included in the article's Creative Commons licence and your intended use is not permitted by statutory regulation or exceeds the permitted use, you will need to obtain permission directly from the copyright holder. To view a copy of this licence, visit http://creativecommons.org/licenses/by/4.0/. The Creative Commons Public Domain Dedication waiver (http://creativeco mmons.org/publicdomain/zero/1.0/) applies to the data made available in this article, unless otherwise stated in a credit line to the data. 
Keywords: Pain, Disability, Steroids, Acupuncture, Manual therapy

\section{Background}

Corticosteroid injections (CSI) have been widely used in the management of musculoskeletal conditions in order to reduce inflammation, pain and disability [1]. Corticosteroids are exogenous drugs that mimic the endogenous steroid hormones and are involved in physiological process such as the regulation of metabolism, skeletal growth and immune function [2]. The anti-inflammatory effects of corticosteroids may be due to a down-regulation of pro-inflammatory cytokines and genes [2]. As inflammation is involved in pain-related mechanisms, corticosteroid injections are useful to reduce pain and, consequently, disability.

Although some studies have found positive effects of corticosteroid injections for common musculoskeletal conditions, other trials have found no clinically meaningful improvements in comparison to placebo injections [3-5]. In addition, corticosteroid injections cause adverse effects [6, 7]. It has been showed that local inflammation may increase up to three days following corticosteroid application, as well as adrenal suppression and cartilage damage [6]. A randomized clinical trial (RCT) observed that an intra-articular corticosteroid injection caused loss of cartilage without reducing pain at two years follow-up [7]. These findings suggest that corticosteroid injections may be used with caution.

An alternative option to the use of corticosteroid injections is dry needling (DN). It consists of a needling stimulation without drugs that can be used in various body areas aiming to reduce pain and disability. Although its mechanisms are not fully understood, it has been suggested that a local twitch response provoked by dry needling may send neural inputs to the brain that would help to break the vicious cycle of pain-spasm-pain $[8,9]$. Dry needling stimulation is suggested to reduce the nociceptive output in different tissues by improving blood flow, increasing fibroblastic activity and modulating central mechanisms [8].

A previous meta-analysis has shown that dry needling is superior to control/sham dry needling for pain and functional outcomes in individuals with musculoskeletal conditions [11]. However, the observed differences were not considered to be clinically meaningful for pain outcomes. Whereas the treatment mean difference for dry needling on pain scores was 1.27 points, an expected clinically meaningful change would be superior to a 2-point change on the Visual Analogue Scale (VAS) [11]. Compared to other treatments such as soft tissue manual therapy interventions, dry needling exhibited better improvements on pain intensity and pain pressure threshold at a 12 weeks follow-up [11]. In addition to its clinical effects, there are reports of side effects following dry needling therapy. Alternatively, a survey with physiotherapists has only reported mild adverse events related to dry needling intervention such as bruising, bleeding and pain during/after treatment [12]. Reports of aggravating symptoms, fatigue, nausea, and numbness were uncommon. Another survey has found similar results [13]. Although minor adverse events were reported in over a third of DN treatment sessions, major adverse events were rare $(<0.1 \%)$ [13].

The effect of corticosteroid injections and dry needling for musculoskeletal conditions varies at different timepoints $[3,5,8,11]$. Most studies have found positive findings for both interventions at short-term follow-up. It has been suggested that corticosteroid injections are more effective at short-term because their effects are associated with the short half-live of the corticosteroids that are injected [14]. In this way, dry needling would be more effective than corticosteroid injections at longer follow-up assessments. Although it is already known that corticosteroid injections and dry needling interventions change pain and disability outcomes in people with musculoskeletal conditions $[3,5,8,11]$, no previous systematic reviews were found in order to summarize and to compare the effects of these interventions at different follow-up periods.

It is relevant to investigate the effects of dry needling and corticosteroid injections for musculoskeletal conditions because these techniques have been used routinely in the primary care regardless of the recommendations from evidence-based clinical practice guidelines. More than $50 \%$ of physiotherapists responding to a survey in the United States reported to use dry needling in their clinical practice [15]. In addition, a previous study showed that steroid injection is the second most used therapy for managing shoulder pain by Australian general practitioners [16]. Despite the frequent use of these therapies, clinical practice guidelines for musculoskeletal conditions have not usually mentioned neither dry needling nor corticosteroid injections as first line treatment [17-21]. Some guidelines have recommended dry needling and corticosteroid injection as adjunct treatment for some musculoskeletal conditions such as plantar fasciitis and Achilles pain, but it is suggested to be used with caution $[18,19,21]$.

Given that (1) both corticosteroid injection and dry needling have been extensively used to manage 
musculoskeletal conditions, (2) it is unclear if one intervention is superior over the other for pain and disability outcomes, and (3) the effect of these interventions at different time points is under investigated, the aim of this systematic review was to compare the effect of these techniques on pain and disability in individuals with musculoskeletal conditions at short-, medium- and longterm follow-up.

\section{Methods}

\section{Eligibility criteria}

This review was registered with PROSPERO (CRD42020148650) and has been reported according to the Preferred Reporting Items for Systematic Reviews and Meta-Analyses (PRISMA) guidelines [22].

Included studies were randomized clinical trials (RCTs), fastidious or pragmatic, that investigated the effects of dry needling compared to corticosteroid injection. Tenotomy was not considered. Participants had to have musculoskeletal pain and to be over 18 years. Musculoskeletal pain was defined as pain located in the muscles, bone, joints, or tendons such as low back and neck pain [23]. This review considered both localized and widespread musculoskeletal pain regardless the duration of pain. The outcome had to be pain and/or disability. Studies using oral corticosteroids (not injectable) were excluded. Pilot studies and conference abstracts were also excluded. Trials using corticosteroid mixture injections and trials allowing co-interventions were included. No language or follow-up restriction was applied.

\section{Information sources and search strategy}

Search was conducted by two independent researchers in the following databases (from earliest record to 31 October 2021): MEDLINE (Pubmed), SCOPUS (Elsevier), CINAHL (EBSCO), SPORTDiscus (EBSCO) and Web of Science (Clarivate analytics). Search strategy included keywords related to 'dry needling' and 'corticosteroids' using Booleans operators. Searches were not peer reviewed. The full search strategy is described in the Appendix (available as Additional file 1). Citation tracking (hand search of the reference list of eligible studies and published relevant reviews) was performed. Grey literature (http://www.opengrey.eu and http://oaister.world cat.org/) and study registers (https://clinicaltrials.gov/) were searched.

\section{Selection process}

The selection of eligible studies was performed by two investigators independently that screened titles, abstracts and full-text articles to achieve a consensus on which studies to select. Only one investigator performed data extraction from the included studies using a self-developed screening form. Then, the second investigator checked the extracted data. A third investigator resolved any disagreement. Extracted information included study characteristics (author, year, condition, funding source, conflict of interest), methods (study design, data collection period, country), participant's characteristics (sample size, sex, age), intervention details (type of intervention, dose and frequency), outcomes measures and time points (follow up), results, adverse events and responder analysis, when reported. When necessary, missing data were requested from study authors.

\section{Risk of bias assessment}

To assess the risk of bias, the revised Cochrane Collaboration tool for assessing risk of bias (Risk of Bias 2.0) in randomized clinical trials was used [24]. This tool evaluates five domains: bias arising from the randomization process, bias due to deviations from intended intervention, bias due to missing outcome data, bias in measurement of the outcome, and bias in selection of the reported result. Two independent investigators made the judgment of each item as at either 'low risk', 'high risk' or 'some concerns'. Any disagreement was resolved by discussion between the investigators. If consensus was not achieved by discussion, a third investigator opinion was sought.

\section{Certainty assessment}

The quality of evidence for each outcome was assessed by using the Grading of Recommendations Assessment, Development and Evaluation (GRADE) approach [25]. Five factors were considered in the overall quality evaluation: risk of bias $(\geq 25 \%$ of the trials were judged as at high risk of bias), inconsistency $\left(\mathrm{I}^{2} \geq 50 \%\right.$ or if pooling was not possible), indirectness $(\geq 50 \%$ of the trials presented differences in study population, interventions or outcome measures), imprecision ( $<400$ participants per outcome) and publication bias (assessed by funnel plot asymmetries whether there are ten studies or more for each outcome). For each factor judged to be present, the quality of evidence was downgraded one level. Overall quality of evidence levels were classified into high, moderate, low or very low quality.

\section{Data synthesis}

Data analysis was based on the follow-up period (shortterm: $\leq 6$ weeks; mid-term: 7-23 weeks; and long-term: $\geq 24$ weeks). Results were expressed as mean \pm SD for each measure. When outcomes scales differed from the direction of the effect, we have multiplied the mean values of the differing scales by -1 . Meta-analysis was not 
performed due to the high level of heterogeneity across studies.

\section{Results}

A total of 1,299 studies were identified from databases in the initial search. After duplicates were removed, 696 remained. Of these, 18 were retrieved for full-text screening. Six studies were included in the final analysis [26-31]. Although 1071 studies were identified from other sources, none was retrieved for full-text screening (Fig. 1).

\section{Study characteristics}

The included studies involved 384 participants with 190 enrolled in DN group and 194 enrolled in CSI group. The characteristics of the studies are shown in Table 1. The majority of the participants were female and the estimated average age was 49 years old. Two studies did not report data for sex of the participants $[29,30]$. Musculoskeletal conditions assessed were plantar fasciitis (2 trials) $[27,28]$, greater trochanteric pain syndrome (1 trial) [26], lateral epicondylitis (2 trials) [30, 31] and myofascial pain with associated headache (1 trial) [29]. These conditions were chronic (symptoms $>3$ months) in four trials. One trial did not limit the duration of symptoms [29]. No conflict of interest was reported.
All studies had pain as an outcome. Three studies investigated disability. One study has used the Patient Reported Tennis Elbow Evaluation (PRTEE) tool, which evaluates both pain and disability [30]. Pain was assessed by using different scales: Foot Function Index (FFI) [28], Visual Analogue Scale (VAS) [27], Numeric Pain Rating Scale (NPRS) [26], and modified Symptom Severity Index (SSI) [29]. Disability was assessed though FFI [28], Disabilities of the Shoulder, Arm and Hand (DASH) [31] and Patient-Specific Functional Scale (PSFS) [26]. Short-term effects were assessed by all studies and medium- and long-term effects were evaluated by five studies [27-31]. Dosage and type of corticosteroid injection varied across the studies. Three studies used a corticosteroid mixture containing anesthetics $[26,28,29]$. For dry needling intervention, the needle diameter and the technique parameters (time left in situ, puncture pattern) were different across the studies.

All studies used one single dose application of corticosteroid injections. For dry needling intervention, Rastegar et al. [27] and Venancio et al. [29] used one single session of treatment, Uygur et al. [28, 30] used 5 sessions (twice a week), Güngör et al. [31] used 3 sessions (once a week) and Brennan et al. [26] used between 3 and 7 sessions (at discretion of the therapist).

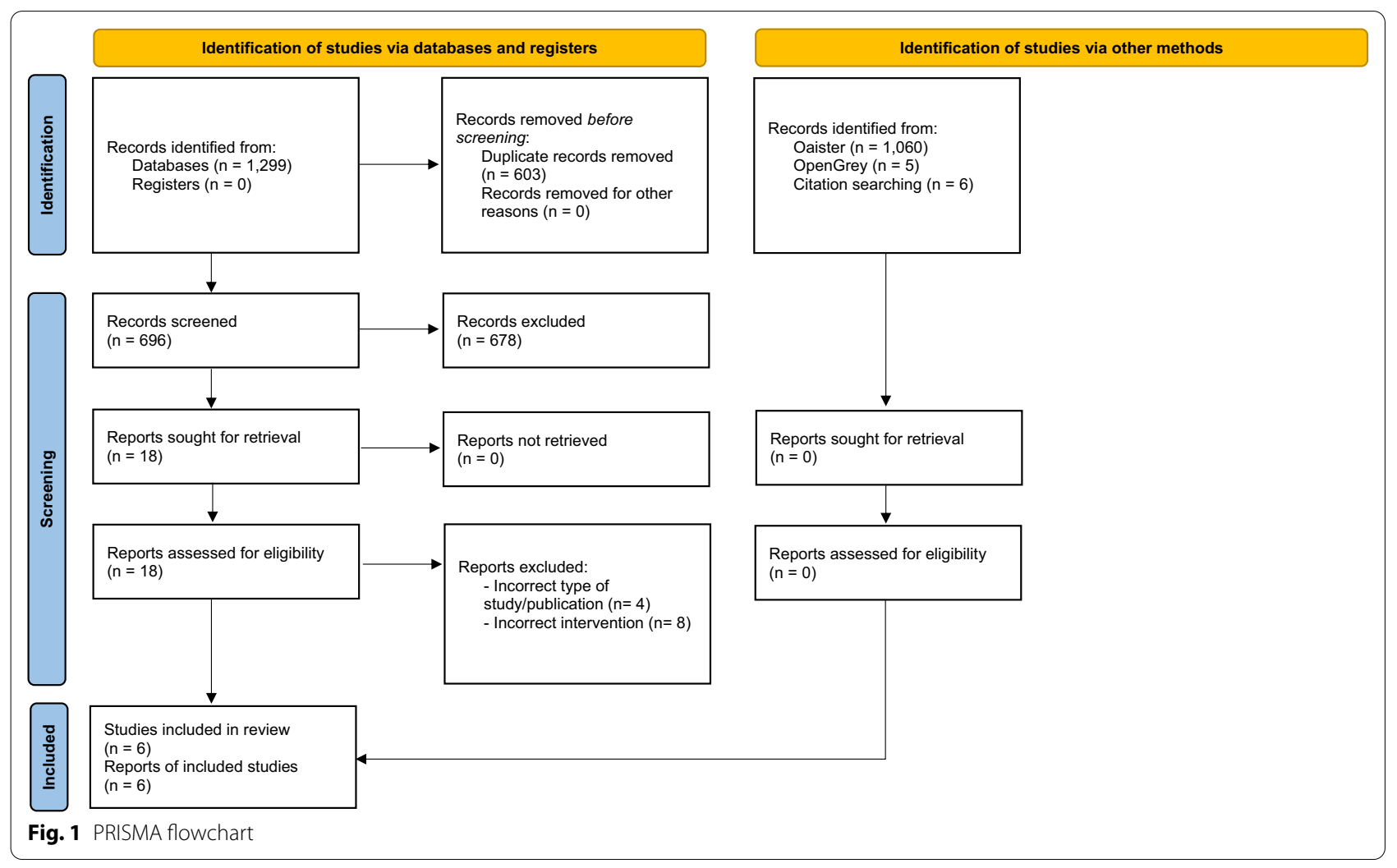




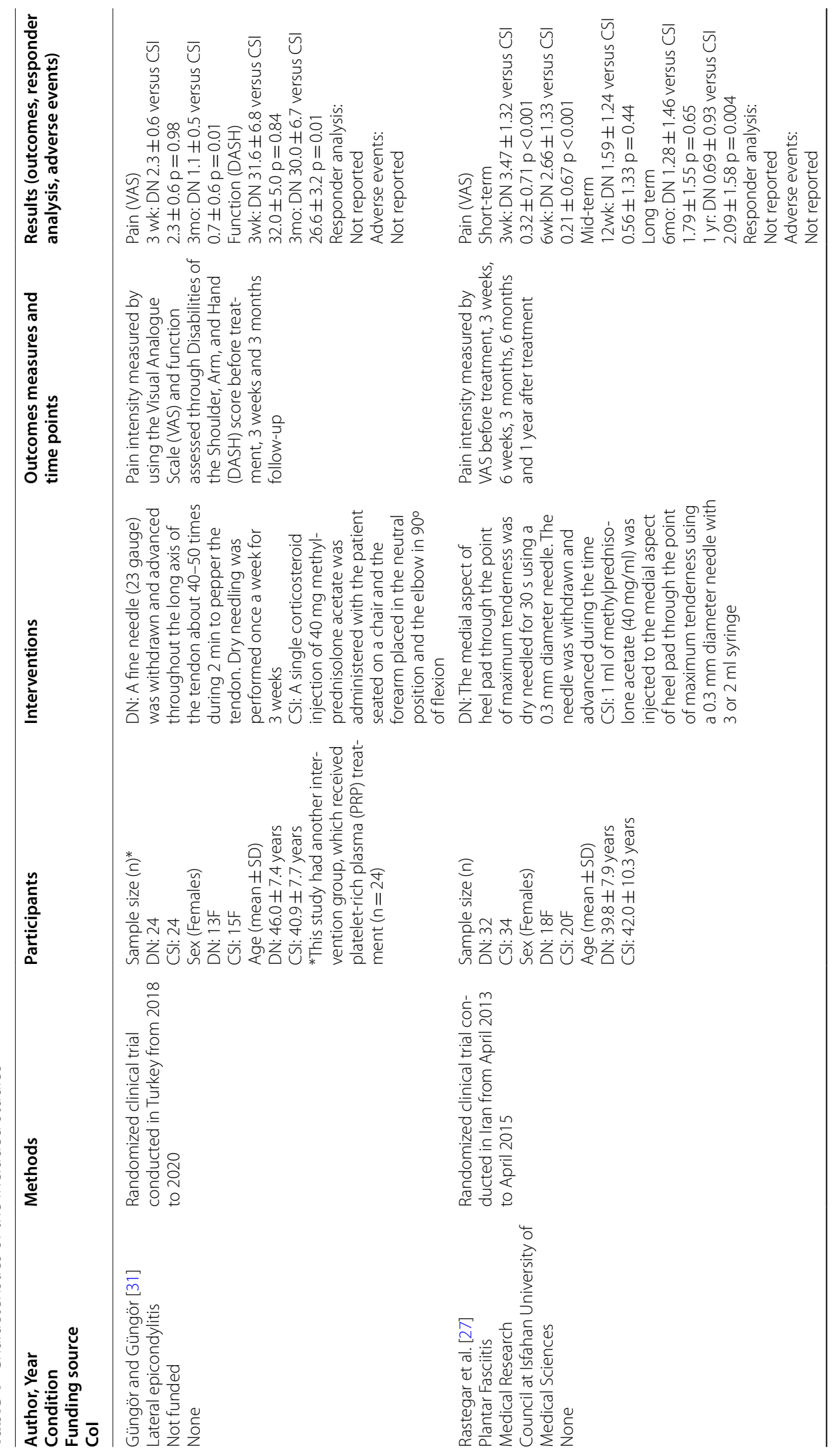




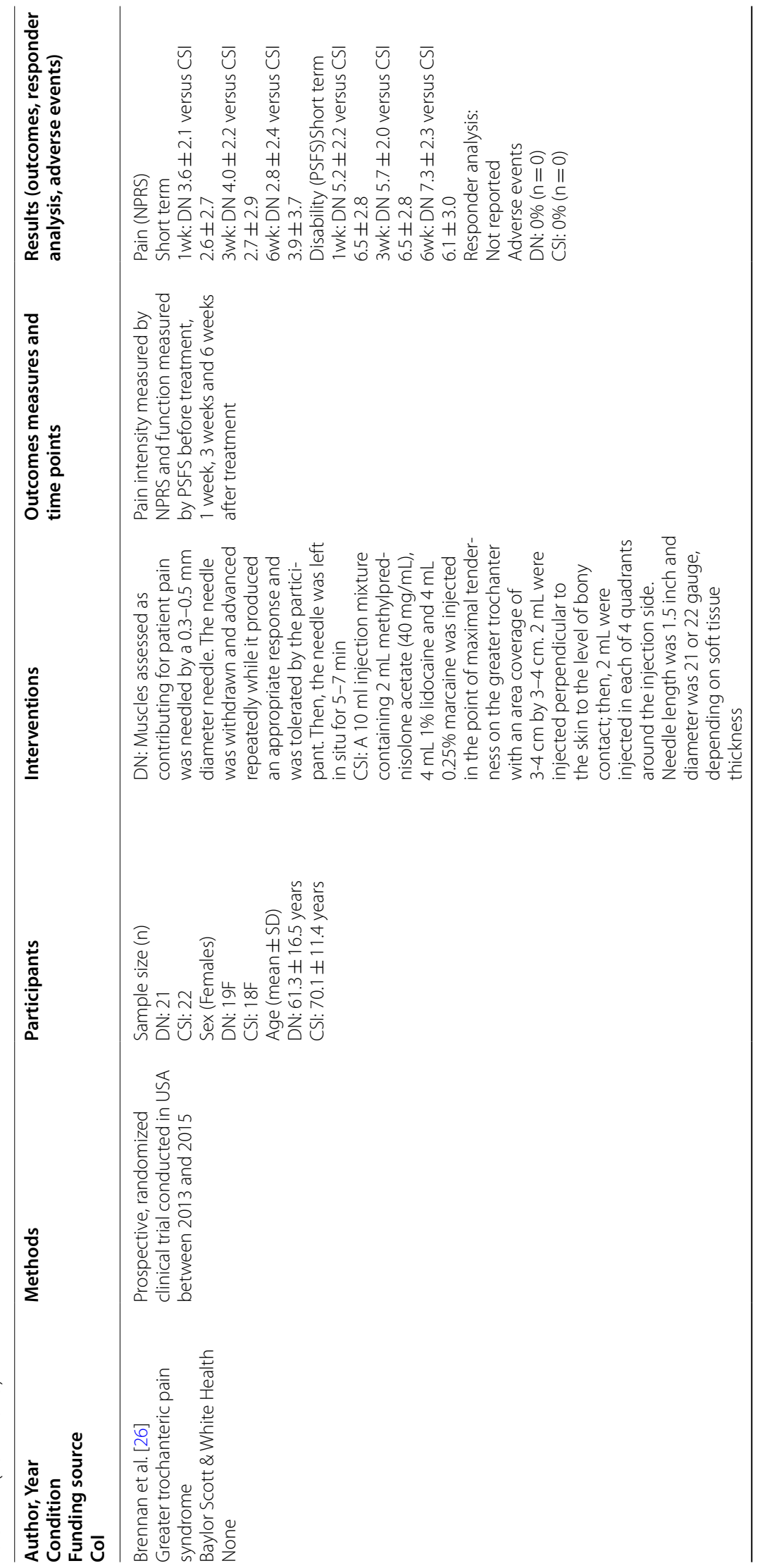




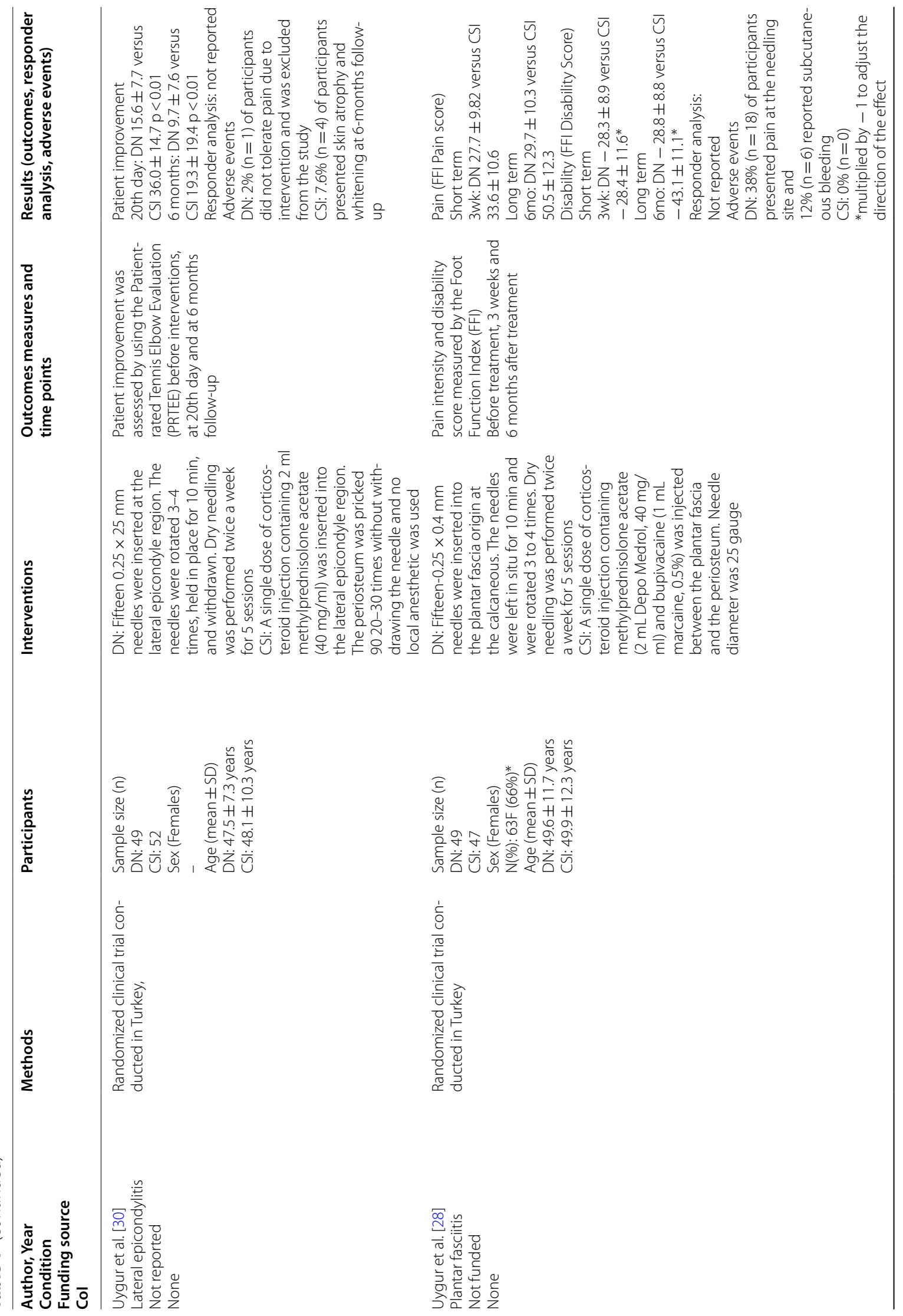




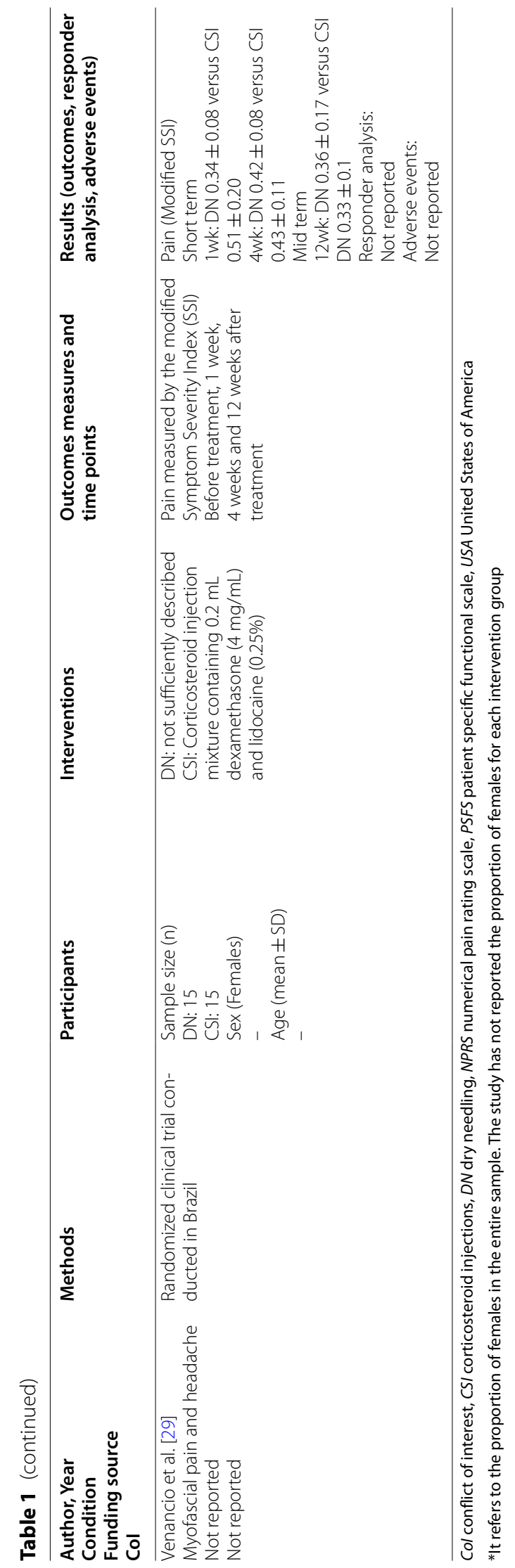




\section{Risk of bias and outcomes}

The risk of bias is shown in Fig. 2. A good interobserver agreement was observed (intraclass correlation coefficient: 0.86) and disagreements were resolved by discussion between first and second investigators. The randomization process was categorized as being at a low risk of bias for one trial [27], at a high risk of bias for two trials $[26,30]$ and with some concerns for three trials $[28,29,31]$. The description of the allocation concealment was unclear for most trials. Four studies exhibited high risk of bias due to deviations from intended intervention [28-31] and two exhibited high risk of bias for missing outcome data [28, 29]. Two studies presented high risk of bias for measurement of the outcome $[29,31]$ and four studies presented some concerns for selection of the reported result [28-31]. Brennan et al. [26] presented an outcome that was not pre-specified in the study registry, which indicates a selective reporting. Two trials presented some bias related to missing sample size calculation along with insufficient study reporting [29] and allowing co-intervention for only one treatment group [28]. Overall, four studies were judged as at a high risk of bias [28-31], one study was considered to present some concerns [26] and another was judged as at low risk of bias [27]. One study was judged as at low risk of bias for all five risk of bias domains [27].

The quality of evidence for each comparison is detailed in Table 2. All studies reported results for the short-term analysis. At 1 week, Brennan et al. [26] (DN $3.6 \pm 2.1$ vs CSI $2.6 \pm 2.7$ ) and Venancio et al. [29] (DN $0.34 \pm 0.08$ vs CSI $0.51 \pm 0.20$ ) have found no difference between DN and CSI for greater trochanteric and myofascial pain/headache, respectively. At 3 weeks, whereas Uygur et al. [28] (3wk: DN $27.7 \pm 9.82$ vs CSI $33.6 \pm 10.6$ ) and Brennan et al. [26] (DN 4.0 \pm 2.2 vs CSI $2.7 \pm 2.9)$ found no significant between-group difference for heel (plantar fasciitis) and greater trochanteric pain, respectively, Rastegar et al. [27] (DN 3.47 \pm 1.32 vs CSI $0.32 \pm 0.71$ ) found more reduction in heel pain (plantar fasciitis) for CSI group. Even although Güngör et al. [31] found no difference between dry needling and corticosteroid injection (DN $2.3 \pm 0.6$ vs CSI $2.3 \pm 0.6$ ) for people with lateral epicondylitis at 3 weeks, Uygur et al. [30] found a significant difference in favor of dry needling at $20^{\text {th }}$ day follow-up (DN $15.7 \pm 7.7$ vs CSI $36.0 \pm 14.7)$. At 4 weeks, Venancio et al. [29] (DN $0.42 \pm 0.08$ vs CSI $0.43 \pm 0.11$ ) found no differences between DN and CSI for myofascial pain/headache. At 6 weeks, Brennan et al. [26] (DN 2.8 \pm 2.4 vs CSI $3.9 \pm 3.7$ ) found no between-groups difference for greater trochanteric pain, but Rastegar et al. [27] (DN $2.66 \pm 1.33$ vs CSI $0.21 \pm 0.67$ ) observed a significant heel pain (plantar fasciitis) reduction in favor of CSI in comparison to DN. The quality of the evidence was rated as very low-quality (GRADE).

Three studies assessed medium-term effects [27, 29, 31]. At 12 weeks, Venancio et al. [29] (DN $0.36 \pm 0.17$ vs CSI DN $0.33 \pm 0.12$ ) found no differences between $\mathrm{DN}$ and CSI for myofascial/headache pain and Rastegar et al. [27] (DN $1.59 \pm 1.24$ vs CSI $0.56 \pm 1.33 \mathrm{p}=0.44$ ) found that CSI is more effective than DN for reducing heel pain (plantar fasciitis). Güngör et al. [31] found a significant difference in favor of corticosteroid injection for people with lateral epicondylitis at 12 weeks follow-up (DN $1.16 \pm 0.5$ vs CSI $0.7 \pm 0.6)$. The quality of the evidence was rated as very low-quality (GRADE).

Three studies reported results for long-term analysis $[27,28,30]$. Two studies were related to heel pain (plantar fasciitis). Uygur et al. [28] have found that DN was superior to CSI for reducing pain at 6 months follow-up (DN $29.7 \pm 10.3$ vs CSI $50.5 \pm 12.3$ ). Although Rastegar et al. [27] found no significant difference between DN and CSI in reducing pain at 6 months follow-up (DN 1.28 \pm 1.46 vs CSI $1.79 \pm 1.55 \mathrm{p}=0.65)$, there was a significant difference in favor of DN at 1-year follow-up (DN $0.69 \pm 0.93$ vs CSI $2.09 \pm 1.58)$. At 6 months follow-up, Uygur et al. [30] found a significant difference in favor of dry needling (DN 9.7 \pm 7.6 vs CSI $19.3 \pm 19.4$ ) for people with lateral epicondylitis. There is very low-quality evidence (GRADE) that DN was superior to CSI in pain reduction at long-term.

For disability, three distinct studies reported data at short term and the quality of evidence was considered to be very low (GRADE) [26, 28, 31]. Brennan et al. [26] evaluated disability in individuals with greater trochanteric pain syndrome at 1 week (DN $5.2 \pm 2.2$ vs CSI $6.5 \pm 2.8$ ), 3 weeks (DN $5.7 \pm 2.0$ vs CSI $6.5 \pm 2.8$ ) and 6 weeks (DN $7.3 \pm 2.3$ vs CSI $6.1 \pm 3.0$ ). No significant between-groups difference was observed. Uygur et al. [28] have also found no significant differences between $\mathrm{DN}$ and CSI (DN $-28.3 \pm 8.9$ vs CSI $-28.4 \pm 11.6)$ for disability in plantar fasciitis at 3 weeks. Güngör et al. [31] assessed disability for people with lateral epicondylitis at 3 weeks and have found no between-group difference between dry needling and corticosteroid injection (DN $31.6 \pm 6.8$ vs CSI $32.0 \pm 5.0)$.

One study presented mid-term results for disability [31]. Corticosteroid injection was superior to dry needling for people with lateral epicondylitis at 3 months follow-up (DN 30.0 \pm 6.7 vs CSI 26.6 \pm 3.2 ) [31]. For the long-term results, Uygur et al. [28] found a greater reduction of disability in DN group compared to CSI group (DN $-28.8 \pm 8.8$ vs CSI $-43.1 \pm 11.1$ ) for individuals with plantar fasciitis at 6 months follow-up. The quality of evidence was rated as very low-quality (GRADE). 


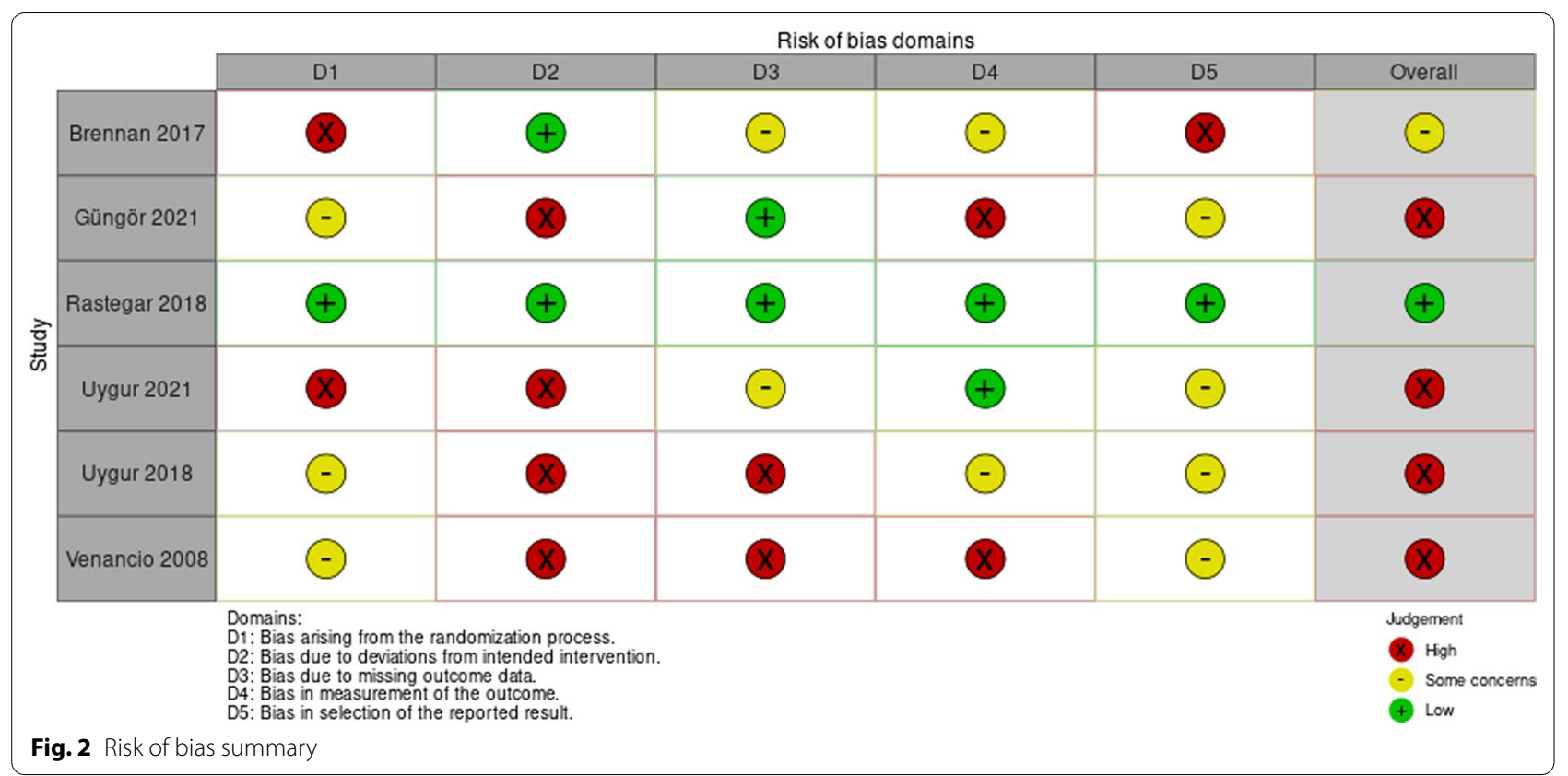

Table 2 Quality of evidence according to GRADE approach

\begin{tabular}{llllllll}
\hline Outcome & Participants (studies) & Risk of bias & Inconsistency & Indirectness & Imprecision & $\begin{array}{l}\text { Publication } \\
\text { bias }\end{array}$ & $\begin{array}{l}\text { Overall } \\
\text { Quality of } \\
\text { evidence }\end{array}$ \\
\hline Pain (short-term) & $384(6$ trials) & Serious & Serious & Serious & Serious & NA & Very low \\
Pain (medium-term) & $144(3$ trials) & Serious & Serious & Not serious & Serious & NA & Very low \\
Pain (long-term) & $263(3$ trials) & Serious & Serious & Serious & Serious & NA & Very low \\
Disability (short-term) & $187(3$ trials) & Serious & Serious & Serious & Serious & NA & Very Low \\
Disability (medium-term) & $48(1$ trial) & Serious & Serious & Serious & Serious & NA & Very low \\
Disability (long-term) & $96(1$ trial) & Serious & Serious & Serious & Serious & NA & Very low \\
\hline
\end{tabular}

NA not applicable

\section{Discussion}

Whereas dry needling and corticosteroid injections appear to present the same effect on pain and disability for people with myofascial pain and greater trochanteric pain syndrome, these interventions are likely to present distinct effects for heel pain (plantar fasciitis) and lateral epicondylitis. At short- and medium-term, corticosteroid injection seems to be superior to dry needling for reducing pain and disability in musculoskeletal conditions. At long-term, dry needling seems to be more effective than corticosteroid injection. However, the quality of evidence behind these findings is very low. In order to improve clinical decision making between dry needling and corticosteroid injection for musculoskeletal conditions, some important considerations regarding our results need to be taken into account.

Most included studies were at high risk of bias, which may affect the strength of the results. Although most studies presented small sample size, five studies calculated sample size for an $\alpha$ level of 0.05 and $80 \%$ power [26-28, 30, 31]. One study did not describe sample size calculation and presented a small sample size, which indicates it is very likely to be underpowered [29]. Evidence from this study was considered to be of poor quality because underpowered trials tend to over-estimate effect sizes [32]. Blinding of participants and personnel and allocation concealment were the main sources of bias. A previous review investigating the effectiveness of dry needling for musculoskeletal conditions has found blinding of therapists and subjects, intention-to-treat analysis and concealed allocation as the main sources of bias [11]. Another meta-analysis observed that the most common sources of bias among the studies verifying the effectiveness of corticosteroid injection for plantar heel pain were blinding of participants/personnel and outcome assessors [33]. While blinding of participants and therapists 
is typically not feasible for studies included in our systematic review due to the nature of interventions, concealed allocation should be adequately implemented and described in order to avoid selection bias. Although some studies did not observe significant influence of allocation concealment on treatment estimates of several interventions [34-36], there is no recommendation to stop using allocation concealment as a bias indicator because other previous works showed that this quality indicator may over- or underestimate the treatment effects $[37,38]$. In addition, inadequate allocation concealment and lack of blinding contribute to an increased between-trial heterogeneity in meta-analyses studies $[39,40]$. The high level of heterogeneity across studies in our systematic review prevented us from conducting a meta-analysis.

Despite the low methodological quality, our results indicate that there is no significant difference between CSI or DN for reducing pain at short-term follow-up ( $\leq 6$ weeks) in individuals with greater trochanteric and myofascial pain/headache $[26,29]$. This finding is intriguing because, if both interventions provide the same effect, clinical decision-making could consider other factors beyond interventions effect on pain such as adverse effects, cost or patient preference. However, the results may be interpreted with caution because the effectiveness of these interventions against placebo is still controversial. At the short-term, one study found effects in favor of corticosteroid injection at 3 weeks and 6 weeks for reducing pain in individuals with plantar fasciitis when compared to dry needling [27]. This study showed outcomes that achieved the minimal clinically important difference (MCID), which is two points out of ten on an 11-points scale for chronic pain conditions [41], and was considered to be at a lower risk of bias than the others were. At the medium-term (7-23 weeks) follow-up, this same study found effects in favor of corticosteroid injection, but these effects were not clinically significant. Whereas one study has found that DN is superior to CSI for lateral epicondylitis at short-term [30], another study has observed no difference between DN and CSI at shortterm and a significant effect in favor of CSI at mediumterm follow-up [31]. Both studies were at high risk of bias. Therefore, we are not sure if the observed overall short- and medium-term effects would remain the same if studies with higher methodological quality were added to the analysis.

At long-term, dry needling seems to be more effective than corticosteroid injection for reducing heel pain and lateral elbow pain. The between-groups difference found in favor of dry needling in the study assessing people with lateral epicondylitis achieved a minimal clinical significance, which is seven points out 100 in the PRTEE tool [30, 42]. Two studies included in this review investigated the effects of DN and CSI for plantar fasciitis [27, 28]. Although Rastegar et al. [27] found a significant difference in favor of $\mathrm{DN}$ at 1-year follow-up, the achieved reduction in pain levels was not clinically important (MCID $<2$ points). Uygur et al. [28] found a significant pain reduction in DN group in comparison to CSI group at 6 months follow-up. This difference achieved the minimal important difference (MID) for people with plantar fasciitis, which is 6.5 points change in the Foot function index (FFI) [43]. While a previous meta-analysis has found that corticosteroid injections are not different from placebo for pain outcomes [34], a RCT has showed that dry needling is superior to sham dry needling in individuals with plantar heel pain [44] at a 12 weeks follow-up. As placebo effect may reduce over the time, especially because most corticosteroid injections are delivered by one single application and dry needling intervention generally is performed in multiple treatment sessions, it is reasonable to hypothesize that dry needling would show better results than corticosteroid injection at longer follow-up periods. In fact, previous trials have found that corticosteroid injection tends to present either similar or greater effects at short-term than long-term for some comparators and no differences at the longterm $[3,34]$. To ensure that the superiority effect from dry needling at long-term is derived from the intervention itself, large RCTs with adequate power and methodological quality may be performed.

For disability, there was no difference between dry needling and corticosteroid injection for greater trochanteric pain syndrome, plantar fasciitis or lateral epicondylitis at short-term. It is important to note that one study investigating people with plantar fasciitis [28] presented effects on disability that are similar to that observed for pain levels, where dry needling appeared to be more effective than corticosteroid injection for reducing disability at longer follow-up periods. The similarity between pain and disability results may be due to the clinical relationship among these variables. It has been observed that reduction in pain levels lead to reduction in disability scores for individuals with musculoskeletal conditions [45]. As cognitive-emotional factors may mediate this response, when musculoskeletal pain intensity is reduced along with improvements in pain acceptance or catastrophizing, the individuals would feel more confident and fearless to perform activities of daily living, thus reducing disability. Although disability is an important outcome, only half of the included studies reported disability as an outcome. Musculoskeletal conditions are the leading cause of disability worldwide. Therefore, it is highly recommended that future studies include disability as an outcome. 
Both corticosteroid injection and dry needling are suggested to reduce pain and disability from distinct mechanisms. While dry needling effects are derived from the needling stimulation over the tissue, corticosteroid injection effects are derived from the action of the drug that is being injected [46]. Dry needling elicits a local twitch response that emits neural signals capable of breaking the vicious cycle of pain-spasm-pain [8, 46]. As there is no consensus regarding needling duration, frequency, location and intensity [8], previous studies have adopted different needling procedures to assess the effects of dry needling for musculoskeletal pain and functional outcomes [11]. In our review, dry needling intervention was applied under different protocols, which would contribute to high heterogeneity across studies. For this reason, meta-analysis was not performed and we were not able to examine to which extent the differences in dry needling application across the studies are likely to influence the outcomes. It is reasonable to suggest that dry needling results could be favored by the increased contact between patient-therapist. Whereas CSI was performed in one single session, DN intervention was delivered in up to 7 sessions. As the patient-therapist interaction may be a source of implicit bias, it may be considered when interpreting the results [47].

Corticosteroid injection action primarily depends on the mechanism of corticosteroid drugs. Usually, these drugs act by suppressing release or activation of proinflammatory cytokines such as interleukins, chemokines and prostaglandins. Three studies in our review have used a particulate steroid (methylprednisolone acetate), while one study used a non-particulate steroid (dexamethasone). Although non-particulate steroids are short-acting and more soluble than particulate steroids, we believe that the type of injected steroid would not influence our findings because the only study that has used non-particulate steroid just assessed short- and medium-term effects [48]. Thus, the effects of the shortacting steroid would be still present in these time points. Ongoing studies may better clarify if there is difference between particulate and non-particulate steroids for common musculoskeletal conditions.

This study has some limitations. Only six studies were included in the review, which limits the comparisons, reduces the strength of the results, and weakens the generalization of the findings. In addition, the treatment estimates presented substantial heterogeneity, which prevented us from conducting a meta-analysis. Furthermore, the methodological quality of the studies was very low quality. Therefore, we emphasize the need for large RCTs with adequate sample size and statistical power as well as adequate study reporting with proper description of allocation concealment, blinding, and intervention procedures.

\section{Conclusions}

In conclusion, there is very low quality of evidence that there is no significant difference between CSI or DN for pain or disability at short-, medium- or long-term follow-up in people with myofascial pain and greater trochanteric pain syndrome. Very low-certainty evidence suggests that corticosteroid injection is superior to dry needling at shorter follow-up and dry needling is more effective than corticosteroid injection at long-term follow-up for reducing pain and/or disability in people with plantar fasciitis and lateral epicondylitis. Large RCTs with higher methodological quality are needed in order to draw more incisive conclusions.

Clinicians and researchers may be aware that although both interventions presented effects for pain at short-, medium-, and long-term follow-up in the assessed musculoskeletal conditions, these findings are supported by insufficient evidence. Therefore, we suggest that corticosteroid injections and dry needling should be used with caution in the clinical settings and when making a clinical decision about which intervention to use, the professionals should also consider other factors such as adherence, costs, patient preference and adverse events. The finding that CSI seems to be more effective than DN at shorter follow-ups and DN appears to be superior to CSI at longterm follow-up for the management of heel and lateral elbow pain and that DN and CSI seems to present similar effects for the management of myofascial and greater trochanteric pain may help clinicians to make informed clinical choices when choosing to use these interventions as adjunct therapies.

\section{Supplementary Information}

The online version contains supplementary material available at https://doi. org/10.1186/s12998-021-00408-y.

Additional file 1. Search strategy.

\section{Acknowledgements}

Not applicable.

\section{Authors' contributions}

LFSF, MMBS, and WMSJ developed the research questions and methodology. LFSF and MMBS conducted the searches in the databases. WMSJ resolved any disagreement in the selection of studies. LFSF and GHFS assessed the risk of bias. LFSF, MMBS, GHFS and WMSJ participated in data analysis and interpretation of results. LFSF, MMBS and GHFS participated in the writing of article. All authors read and approved the final manuscript.

\section{Funding}

The authors received no financial support for the research, authorship, and/or publication of this article. 


\section{Availability of data and materials}

All data generated or analyzed during this study are included in this published article [and its Additional file 1].

\section{Declarations}

\section{Ethics approval and consent to participate}

Not applicable.

\section{Consent for publication}

Not applicable.

\section{Competing interests}

The authors declare that they have no competing interests.

\section{Author details}

${ }^{1}$ Graduate Program in Physical Education, Federal University of Sergipe, Av Marechal Rondon, s/n, Rosa Elze, São Cristovão, Sergipe 49100-000, Brazil. ${ }^{2}$ Department of Physical Therapy, Federal University of Sergipe, Av Marechal Rondon, s/n, Rosa Elze, São Cristovão, Sergipe 49100-000, Brazil.

Received: 10 June 2021 Accepted: 22 November 2021

Published online: 02 December 2021

\section{References}

1. Stephens MB, Beutler Al, O'Connor FG. Musculoskeletal injections: a review of the evidence. Am Fam Physician. 2008;78(8):971-6.

2. Ramamoorthy $S$, Cidlowski JA. Corticosteroids: mechanisms of Action in Health and Disease. Rheum Dis Clin N Am. 2016:42(1):15-vii.

3. Li Z, Yu A, Qi B, et al. Corticosteroid versus placebo injection for plantar fasciitis: a meta-analysis of randomized controlled trials. Exp Ther Med. 2015;9(6):2263-8.

4. Claessen F, Heesters BA, Chan JJ, et al. A meta-analysis of the effect of corticosteroid injection for enthesopathy of the extensor carpi radialis brevis origin. J Hand Surg. 2016;41(10):988-998.e2.

5. Wang W, Shi M, Zhou C, et al. Effectiveness of corticosteroid injections in adhesive capsulitis of shoulder: a meta-analysis. Medicine. 2017;96(28):e7529.

6. Freire V, Bureau NJ. Injectable corticosteroids: take precautions and use caution. Semin Musculoskel R. 2016:20(5):401-8.

7. McAlindon TE, LaValley MP, Harvey WF, et al. Effect of intra-articular triamcinolone vs saline on knee cartilage volume and pain in patients with knee osteoarthritis: a randomized clinical trial. JAMA. 2017:317(19):1967-75.

8. Dunning J, Butts R, Mourad F, et al. Dry needling: a literature review with implications for clinical practice guidelines. Phys Ther Rev. 2014;19(4):252-65.

9. Cagnie B, Dewitte V, Barbe T, et al. Physiologic effects of dry needling. Curr Pain Headache R. 2013;17(8):348.

10. Dommerholt J. Dry needling - peripheral and central considerations. J Man Manip Ther. 2011;19(4):223-7.

11. Gattie E, Cleland JA, Snodgrass S. The effectiveness of trigger point dry needling for musculoskeletal conditions by physical therapists: a systematic review and meta-analysis. J Orthop Sport Phys. 2017:47(3):133-49.

12. Brady S, McEvoy J, Dommerholt J, et al. Adverse events following trigger point dry needling: a prospective survey of chartered physiotherapists. J Man Manip Ther. 2014;22(3):134-40.

13. Boyce D, Wempe H, Campbell C, Fuehne S, Zylstra E, Smith G, Wingard C, Jones R. Adverse events associated with therapeutic dry needling. Int J Sports Phys Ther. 2020;15(1):103-13.

14. Wehling P, Evans C, Wehling J, Maixner W. Effectiveness of intra-articular therapies in osteoarthritis: a literature review. Ther Adv Musculoskelet Dis. 2017;9(8):183-96.

15. Gattie E, Cleland JA, Snodgrass S. A survey of American physical therapists' current practice of dry needling: practice patterns and adverse events. Musculoskelet Sci Pract. 2020:50:102255.
16. Naunton J, Harrison C, Britt H, Haines T, Malliaras P. General practice management of rotator cuff related shoulder pain: a reliance on ultrasound and injection guided care. PLOS ONE. 2020;15(1):e0227688.

17. Lin I, Wiles L, Waller R, Goucke R, Nagree Y, Gibberd M, Straker L, Maher CG, O'Sullivan PPB. What does best practice care for musculoskeletal pain look like? Eleven consistent recommendations from high-quality clinical practice guidelines: systematic review. Br J Sports Med. 2020;54(2):79-86.

18. Morrissey D, Cotchett M, Said J'Bari A, Prior T, Griffiths IB, Rathleff MS, Gulle H, Vicenzino B, Barton CJ. Management of plantar heel pain: a best practice guide informed by a systematic review, expert clinical reasoning and patient values. Br J Sports Med. 2021;55(19):1106-18.

19. Schneider HP, Baca JM, Carpenter BB, Dayton PD, Fleischer AE, Sachs BD. American college of foot and ankle surgeons clinical consensus statement: diagnosis and treatment of adult acquired infracalcaneal heel pain. J Foot Ankle Surg. 2018;57(2):370-81. https://doi.org/10.1053/j.jfas.2017. 10.018.

20. Martin RL, Chimenti R, Cuddeford T, Houck J, Matheson JW, McDonough CM, Paulseth S, Wukich DK, Carcia CR. Achilles pain, stiffness, and muscle power deficits: midportion achilles tendinopathy revision 2018. J Orthop Sports Phys Ther. 2018;48(5):A1-38.

21. de Vos RJ, van der Vlist AC, Zwerver J, Meuffels DE, Smithuis F, van Ingen R, van der Giesen F, Visser E, Balemans A, Pols M, Veen N, den Ouden M, Weir A. Dutch multidisciplinary guideline on Achilles tendinopathy. $\mathrm{Br} J$ Sports Med. 2021;29:bjsports-2020-103867.

22. Page MJ, McKenzie JE, Bossuyt PM, Boutron I, Hoffmann TC, Mulrow CD, et al. The PRISMA 2020 statement: an updated guideline for reporting systematic reviews. BMJ. 2021:372:71.

23. Nicholas M, Vlaeyen JWS, Rief W, Barke A, Aziz Q, Benoliel R, Cohen M, Evers S, Giamberardino MA, Goebel A, Korwisi B, Perrot S, Svensson P, Wang SJ, Treede RD. IASP Taskforce for the Classification of Chronic Pain. The IASP classification of chronic pain for ICD-11: chronic primary pain. Pain. 2019;160(1):28-37.

24. Sterne JAC, Savović J, Page MJ, Elbers RG, Blencowe NS, Boutron I, Cates CJ, Cheng HY, Corbett MS, Eldridge SM, Emberson JR, Hernán MA, Hopewell S, Hróbjartsson A, Junqueira DR, Jüni P, Kirkham JJ, Lasserson T, Li T, McAleenan A, Reeves BC, Shepperd S, Shrier I, Stewart LA, Tilling K, White IR, Whiting PF, Higgins JPT. RoB 2: a revised tool for assessing risk of bias in randomised trials. BMJ. 2019;366:14898.

25. Guyatt G, Oxman AD, Akl EA, Kunz R, Vist G, Brozek J, Norris S, Falck-Ytter Y, Glasziou P, DeBeer H, Jaeschke R, Rind D, Meerpohl J, Dahm P, Schünemann HJ. GRADE guidelines: 1. Introduction-GRADE evidence profiles and summary of findings tables. J Clin Epidemiol. 2011;64(4):383-94.

26. Brennan $\mathrm{KL}$, Allen BC, Maldonado YM. Dry needling versus cortisone injection in the treatment of greater trochanteric pain syndrome: a noninferiority randomized clinical trial. J Orthop Sport Phys. 2017;47(4):232-9.

27. Rastegar S, Baradaran Mahdavi S, Hoseinzadeh B, et al. Comparison of dry needling and steroid injection in the treatment of plantar fasciitis: a single-blind randomized clinical trial. Int Orthop. 2018;42(1):109-16.

28. Uygur E, Aktaş B, Eceviz E, et al. Preliminary report on the role of dry needling versus corticosteroid injection, an effective treatment method for plantar fasciitis: a randomized controlled trial. J Foot Ankle Surg. 2019;58(2):301-5

29. Venâncio R, Alencar FG, Zamperini C. Different substances and dryneedling injections in patients with myofascial pain and headaches. Cranio. 2008;26(2):96-103.

30. Uygur E, Aktaş B, Yilmazoglu EG. The use of dry needling vs. corticosteroid injection to treat lateral epicondylitis: a prospective, randomized, controlled study. J Shoulder Elbow Surg. 2021;30(1):134-9.

31. Güngör E, Karakuzu GZ. Comparison of the efficacy of corticosteroid, dry needling, and PRP application in lateral epicondylitis. Eur J Orthop Surg Traumatol. 2021. https://doi.org/10.1007/s00590-021-03138-2.

32. Button KS, loannidis JP, Mokrysz C, Nosek BA, Flint J, Robinson ES, Munafò MR. Power failure: why small sample size undermines the reliability of neuroscience. Nat Rev Neurosci. 2013;14(5):365-76. doi: https://doi.org/ 10.1038/nrn3475. Erratum in: Nat Rev Neurosci. 2013;14(6):451.

33. Whittaker GA, Munteanu SE, Menz HB, et al. Corticosteroid injection for plantar heel pain: a systematic review and meta-analysis. BMC Musculoskel Dis. 2019;20(1):378.

34. Armijo-Olivo S, Saltaji $\mathrm{H}$, da Costa BR, et al. What is the influence of randomisation sequence generation and allocation concealment on 
treatment effects of physical therapy trials? A meta-epidemiological study. BMJ Open. 2015;5(9):e008562.

35. de Almeida MO, Saragiotto BT, Maher C, et al. Allocation concealment and intention-to-treat analysis do not influence the treatment effects of physical therapy interventions in low back pain trials: a meta-epidemiologic study. Arch Phys Med Rehab. 2019;100(7):1359-66.

36. Nüesch E, Reichenbach $\mathrm{S}$, Trelle $\mathrm{S}$, et al. The importance of allocation concealment and patient blinding in osteoarthritis trials: a meta-epidemiologic study. Arthritis Rheum. 2009;61:1633-41.

37. Paludan-Müller A, Teindl Laursen DR, Hróbjartsson A. Mechanisms and direction of allocation bias in randomised clinical trials. BMC Med Res Methodol. 2016;16(1):133.

38. Savović J, Jones HE, Altman DG, et al. Influence of reported study design characteristics on intervention effect estimates from randomized, controlled trials. Ann Inter Med. 2012;157(6):429-38.

39. Rhodes KM, Turner RM, Savović J, et al. Between-trial heterogeneity in meta-analyses may be partially explained by reported design characteristics. J Clin Epidemiol. 2018;95:45-54.

40. Farrar JT, Young JP Jr, LaMoreaux L, Werth JL, Poole MR. Clinical importance of changes in chronic pain intensity measured on an 11-point numerical pain rating scale. Pain. 2001;94(2):149-58.

41. Poltawski L, Watson T. Measuring clinically important change with the Patient-rated Tennis Elbow Evaluation. Hand Therapy. 2011;16(3):52-7. https://doi.org/10.1258/ht.2011.011013.

42. Mischke JJ, Jayaseelan DJ, Sault JD, Emerson Kavchak AJ. The symptomatic and functional effects of manual physical therapy on plantar heel pain: a systematic review. J Man Manip Ther. 2017:25(1):3-10.

43. Cotchett MP, Munteanu SE, Landorf KB. Effectiveness of trigger point dry needling for plantar heel pain: a randomized controlled trial. Phys Ther. 2014;94(8):1083-94.

44. Shaygan M, Böger A, Kröner-Herwig B. How does reduction in pain lead to reduction in disability in patients with musculoskeletal pain? J Pain Res. 2019:12:1879-90.

45. Chou LW, Kao MJ, Lin JG. Probable mechanisms of needling therapies for myofascial pain control. Evid-based Compl Alt. 2012; 705327.

46. FitzGerald C, Hurst S. Implicit bias in healthcare professionals: a systematic review. BMC Med Ethics. 2017;18(1):19. https://doi.org/10.1186/ s12910-017-0179-8.

47. Knezevic NN, Jovanovic F, Voronov D, et al. Do corticosteroids still have a place in the treatment of chronic pain? Front Pharmacol. 2018;9:1229.

\section{Publisher's Note}

Springer Nature remains neutral with regard to jurisdictional claims in published maps and institutional affiliations.

Ready to submit your research? Choose BMC and benefit from:

- fast, convenient online submission

- thorough peer review by experienced researchers in your field

- rapid publication on acceptance

- support for research data, including large and complex data types

- gold Open Access which fosters wider collaboration and increased citations

- maximum visibility for your research: over $100 \mathrm{M}$ website views per year

At BMC, research is always in progress.

Learn more biomedcentral.com/submissions 